\title{
Evidence for Complex Binding Profiles and Species Differences at the Translocator Protein (TSPO) (18 kDa)
}

\author{
A.M. Scarf ${ }^{1,2,3}$, C. Luus ${ }^{1}$, E. Da Pozzo ${ }^{4}$, S. Selleri ${ }^{5}$, C. Guarino ${ }^{5}$, C. Martini ${ }^{4}$, L.M. Ittner ${ }^{3}$ and \\ M. Kassiou ${ }^{\star 2,6,7}$ \\ ${ }^{1}$ Discipline of Pharmacology, University of Sydney NSW 2006, Australia \\ ${ }^{2}$ Drug Discovery Laboratory, Brain and Mind Research Institute, University of Sydney NSW 2050, Australia \\ ${ }^{3}$ Alzheimer's and Parkinson's Disease Laboratory, Brain and Mind Research Institute, University of Sydney \\ NSW 2050, Australia \\ ${ }^{4}$ Department of Psychiatry, Neurobiology, Pharmacology and Biotechnology, University of Pisa, Italy \\ ${ }^{5}$ Department of Pharmaceutical Sciences, University of Florence, Italy \\ ${ }^{6}$ School of Chemistry, University of Sydney NSW 2006, Australia \\ ${ }^{7}$ Discipline of Medical Radiation Sciences, University of Sydney NSW 2006, Australia
}

\begin{abstract}
The translocator protein (TSPO) $(18 \mathrm{kDa})$ is an emerging drug target for the treatment of numerous pathologies including cancer and neurodegenerative disease. However, our limited knowledge of TSPO binding site(s) has hindered the development of TSPO ligands with potential therapeutic effects. We have synthesized a series of pyrrolobenzoxazepines (1-10) to better characterize the interaction of ligands with the TSPO across species, and to determine their functional profiles. All ligands 1-10 displaced the binding of $\left[{ }^{3} \mathrm{H}\right] \mathrm{PK} 11195$ to the TSPO at nanomolar concentrations, with discrepancies in binding affinity between rat and human TSPO. Interestingly, non-linear regression analysis revealed that some ligands bound to the protein with a Hill slope not equal to 1.0, suggesting possible additional TSPO binding sites with allosteric effects. However, this trend was not conserved between rat and human. When tested for their effects on pregnenolone production in rat $\mathrm{C} 6$ glioma cells, nitric oxide release in murine microglia, and cell proliferation in human MCF-7 breast cancer cells, the pyrrolobenzoxazepines $(40 \mu \mathrm{M})$ displayed functional effects which did not correlate to the binding trend observed in competition assays. We propose that consideration of species differences and binding site cooperativity, plus optimization of currently accepted functional assays, will aid in the development of drugs targeting TSPO that can be used as therapeutics for human disease.
\end{abstract}

Keywords: Binding cooperativity, neuroprotection, pyrrolobenzoxazepines, radioligand binding, structure activity relationships, translocator protein (TSPO).

\section{INTRODUCTION}

The TSPO is primarily found in mitochondrial membranes, where its best characterized role is the rate-limiting regulation of steroid biosynthesis. Cholesterol transport from the outer to inner mitochondrial membrane and subsequent conversion to pregnenolone is activated upon ligand binding to the TSPO [1]. Hence, measurement of pregnenolone production is used routinely for the functional evaluation of new TSPO ligands. However, the TSPO has been implicated in other complex cellular functions such as mediating apoptotic and necrotic cell death [1]. Additionally, the TSPO is highly expressed in both neurodegenerative diseases and cancer [1, 2], with protein levels correlated to microglial activation and aggressive metastatic behavior [3]. TSPO ligands exercise both neuroprotective and anti-tumor effects in

*Address correspondence to this author at the Alzheimer's and Parkinson's Disease Laboratory, Brain and Mind Research Institute, University of Sydney NSW 2050, Australia; Tel: +61-2-9351-0849; Fax: +61-2-9351-9146; E-mail: michael.kassiou@sydney.edu.au these pathologies, by inhibiting the production and release of inflammatory mediators from activated microglia [4], and by causing cell-cycle arrest or induction of apoptosis $[5,6]$, respectively. Thus, the TSPO is implicated in both the development of disease and as a drug target for treatment $[1,2,5]$.

Two ligands have been essential for characterizing the TSPO; the benzodiazepine Ro 5-4864 and the isoquinoline carboxamide PK 11195, both of which are selective for the TSPO and display nanomolar binding affinities [7-9]. While these ligands display saturable binding and reciprocal competition in radioligand binding assays $[7,9,10]$, this is not consistent across species [11] and can be modified separately in both rat and human [10]. Furthermore, site-directed mutagenesis studies suggest certain residues in the first putative loop of TSPO are important for the binding of Ro 5-4864, but not PK 11195 [12]. These findings prompted the theory of binding site heterogeneity and/or multiple binding sites at TSPO, the implications of which have not been thoroughly explored. Consequently, while a range of structurally diverse synthetic and semisynthetic TSPO ligands have been 
developed, thorough characterization of their interaction with the TSPO protein is lacking.

To better understand the interaction of ligands with TSPO, we synthesized ten pyrrolobenzoxazepines (110) (Table 1) based on previously reported structureactivity relationships [13]. We screened these ligands for affinity against ${ }^{3} \mathrm{H}$ ]PK 11195 in competitive binding assays in both rat and human TSPO. In addition, we analyzed the curves by non-linear regression to yield a Hill slope value, whereby a slope not equal to 1.0 indicates more than a simple competitive binding interaction, suggesting the possibility of additional sites or allosteric modulation. Additionally, we measured three therapeutically relevant functional parameters, including the ability of the ligands to modulate pregnenolone and nitric oxide (NO) release, and antiproliferative effects, in an attempt to draw correlations between the binding interaction of the ligands with TSPO and the downstream functional consequences.

\section{RESULTS AND DISCUSSION}

In the present study, we evaluated the binding affinity, Hill slopes, and functional effects of ten new pyrrolobenzoxazepines as ligands for the TSPO. Furthermore, to the best of our knowledge, this is the first time this class of compounds has been tested for binding affinity for human TSPO. This study also emphasizes the importance of considering the concept of possible alternate binding sites in routine assays for the screening of new TSPO ligands.

The affinity of synthesized pyrrolobenzoxazepines for TSPO was determined by competition binding assays against $\left[{ }^{3} \mathrm{H}\right] \mathrm{PK}$ 11195, using mitochondrial fractions from rat kidney and human embryonic kidney (HEK293) cells. Subtle structural differences dramatically altered the binding profile of compounds 110. Significant differences in binding affinity between rat and human TSPO were also revealed (Table 1). Accordingly, compound 1 showed a 5.3-fold higher binding affinity in human than in rat, while compounds 4, 6-8 and 10 showed a much higher binding affinity in rat than in human (4: 67.6-fold; 6: 5.6-fold; 7: 5.6-fold; 8: 412.1-fold; 10: 24.1-fold). Compounds 2-3, 5 and 9 presented with similar binding affinities in rat and human. For comparison, cold PK 11195 had a similar binding affinity in rat and human cells, while Ro 5-4864 displayed a 15.7-fold higher binding affinity in rat than in human cells, consistent with previous studies [14]. These findings are of particular interest, since the TSPO is highly conserved, with an amino acid homology of $80 \%$ between rat and human [12]. Thus, the subtle structural differences between rat and human TSPO have a significant impact on the residues

Table 1. General Structure and Cross-Species Binding Trends for the Compounds Synthesized

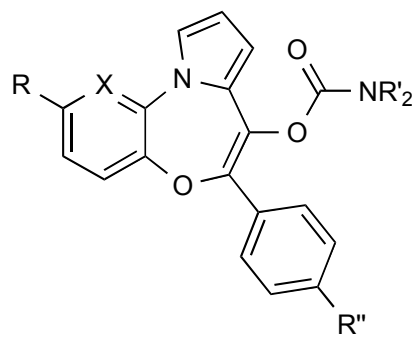

\begin{tabular}{|c|c|c|c|c|c|c|c|c|}
\hline Compound & $x$ & $\mathbf{R}$ & $\mathbf{R}^{\prime}$ & R" & $\begin{array}{l}\mathrm{K}_{\mathrm{i}} \mathrm{nM} \\
\text { (rat) }^{a}\end{array}$ & $\underset{\text { (human) }^{\mathbf{K}_{\mathbf{i}} \mathbf{n M}}}{ }$ & $\begin{array}{l}\text { Hill slope } \\
\text { (rat) }\end{array}$ & $\begin{array}{l}\text { Hill slope } \\
\text { (human) }\end{array}$ \\
\hline 1 & $\mathrm{~N}$ & $\mathrm{H}$ & $\mathrm{CH}_{3}$ & $\mathrm{OCH}_{3}$ & $68.52 \pm 2.0$ & $12.93 \pm 1.41$ & $0.99 \pm 0.09$ & $0.66 \pm 0.05^{* * *}$ \\
\hline 2 & $N$ & $\mathrm{H}$ & $\mathrm{CH}_{2} \mathrm{CH}_{3}$ & $\mathrm{OCH}_{3}$ & $0.43 \pm 0.04$ & $0.91 \pm 0.08$ & $0.75 \pm 0.05$ * & $1.11 \pm 0.08$ \\
\hline 3 & $\mathrm{CH}$ & $\mathrm{H}$ & $\mathrm{CH}_{3}$ & $\mathrm{OCH}_{3}$ & $1.50 \pm 0.10$ & $0.89 \pm 0.08$ & $0.93 \pm 0.08$ & $0.73 \pm 0.05^{* * *}$ \\
\hline 4 & $\mathrm{CH}$ & $\mathrm{H}$ & $\mathrm{CH}_{2} \mathrm{CH}_{3}$ & $\mathrm{OCH}_{3}$ & $0.45 \pm 0.06$ & $30.41 \pm 2.72$ & $2.34 \pm 0.15^{* \star *}$ & $2.11 \pm 0.50^{* * *}$ \\
\hline 5 & $\mathrm{CH}$ & $\mathrm{H}$ & $\mathrm{CH}_{3}$ & $\mathrm{~F}$ & $0.33 \pm 0.03$ & $0.55 \pm 0.06$ & $1.09 \pm 0.12$ & $1.07 \pm 0.12$ \\
\hline 6 & $\mathrm{CH}$ & $\mathrm{H}$ & $\mathrm{CH}_{2} \mathrm{CH}_{3}$ & $\mathrm{~F}$ & $0.40 \pm 0.05$ & $2.26 \pm 0.34$ & $1.03 \pm 0.08$ & $0.93 \pm 0.11$ \\
\hline 7 & $\mathrm{CH}$ & $\mathrm{Cl}$ & $\mathrm{CH}_{3}$ & $\mathrm{OCH}_{3}$ & $9.69 \pm 0.80$ & $54.44 \pm 4.30$ & $1.41 \pm 0.13^{* * *}$ & $1.15 \pm 0.10$ \\
\hline 8 & $\mathrm{CH}$ & $\mathrm{Cl}$ & $\mathrm{CH}_{2} \mathrm{CH}_{3}$ & $\mathrm{OCH}_{3}$ & $0.14 \pm 0.01$ & $57.69 \pm 5.33$ & $1.29 \pm 0.09$ ** & $0.91 \pm 0.08$ \\
\hline 9 & $\mathrm{CH}$ & $\mathrm{CH}_{3}$ & $\mathrm{CH}_{3}$ & $\mathrm{OCH}_{3}$ & $22.19 \pm 2.0$ & $26.19 \pm 2.09$ & $0.99 \pm 0.08$ & $1.17 \pm 0.10$ \\
\hline 10 & $\mathrm{CH}$ & $\mathrm{CH}_{3}$ & $\mathrm{CH}_{2} \mathrm{CH}_{3}$ & $\mathrm{OCH}_{3}$ & $0.68 \pm 0.05$ & $16.39 \pm 1.95$ & $0.84 \pm 0.07$ & $0.77 \pm 0.07^{* *}$ \\
\hline PK 11195 & & & & & $9.3 \pm 0.50$ & $7.11 \pm 0.35$ & $1.01 \pm 0.11$ & $0.99 \pm 0.05$ \\
\hline Ro 5-4864 & & & & & $23.0 \pm 3.0$ & $360.7 \pm 23.62$ & $0.99 \pm 0.10$ & $0.87 \pm 0.07$ \\
\hline
\end{tabular}

${ }^{a} K_{i}$ values represent binding affinities from competition binding experiments against $\left.{ }^{3} \mathrm{H}\right] \mathrm{PK} 11195$ in either rat kidney or HEK293 cells. Values represent the mean \pm SEM of at least three independent experiments carried out in duplicate.

${ }^{*} p<0.05^{* *} p<0.01 ;{ }^{* *} p<0.001$, significantly different to a one-site competition curve will Hill slope equal to 1.0 using extra sum-of-squares $\mathrm{F}$ test. 
involved in ligand binding, and possibly also functional activity across species.

Non-linear regression analysis of $\left[{ }^{3} \mathrm{H}\right] \mathrm{PK} 11195$ displacement in both HEK293 cells and rat kidney revealed more than a simple competitive binding interaction with $\left[{ }^{3} \mathrm{H}\right] \mathrm{PK}$ 11195. While some ligands 1-10 bound with a slope equal to 1.0 (Table 1), indicating competitive binding to a single population of sites, other ligands bound with a slope less or greater than 1.0 (Table 1), denoting a more complex binding profile. Possibilities include binding to multiple sites with different affinities, or negative/positive allosteric interactions. However, different Hill slope values were observed for most compounds across rat and human TSPO (Table 1), again suggesting that binding differs significantly between the two species.

Analysis of competition curves has previously been undertaken for the endogenous TSPO ligand protoporphyrin IX (PPIX), which is able to displace $\left[{ }^{3} \mathrm{H}\right]$ PK 11195 with a slope of 2.0 [15]. Interestingly, PPIX binding is also dose-dependently associated with TSPO dimer formation [15]. Thus, it is plausible to suggest that 4 in human, and 4, 7-8 in rat, with a similar slope, drive the formation of the TPSO dimer which may be responsible for an active form of the protein $[16,17]$. Alternatively, a Hill slope of 2.0 may indicate multiple binding sites involved in positive allosteric modulation. We believe that further research into binding site cooperativity will provide a better understanding of structure-activity relationships at TSPO. While this study revealed no clear structural requirements to account for the variation in slope, this may be due to the small number of compounds in this series.

In addition to displacement of $\left[{ }^{3} \mathrm{H}\right] \mathrm{PK}$ 11195, three functional readouts of the TSPO ligands 1-10 were assessed. Firstly, the induction of pregnenolone release from rat $\mathrm{C} 6$ glioma cells, secondly NO release from murine BV2 microglial cells and thirdly antiproliferative effects in MCF-7 human breast cancer cells. PK 11195 stimulated pregnenolone release from rat C6 glioma cells $37 \pm 1 \%(p<0.001)$ above baseline levels of vehicle treated control (Fig. 1), consistent with previous reports [18]. Interestingly, despite having the lowest binding affinity of all tested compounds to rat TSPO (Table 1), compound 1 was the most potent stimulator of pregnenolone release $(106 \pm 18 \%$ vs. control; $p<0.0001)$. Compounds $2-3$ and 10 had similar effects on pregnenolone release to PK 11195, while 7$8(p<0.01)$ and $9 \quad(p<0.05)$ affected pregnenolone release significantly less than PK 11195. Compounds 4-6 had no effect on pregnenolone release. Thus, the pyrrolobenzoxazepines display a variety of effects on pregnenolone release, at a range consistent with previous studies using this class of compounds [13].

The TSPO has been shown to regulate NO release from activated microglia [4], giving rise to a number of ligands with potential neuroprotective effects. Thus, we determined the amounts of NO released from activated murine BV2 microglial cells upon treatment with PK 11195, 2-3, 5 and 9. Consistent with previous reports [4], PK 11195 significantly inhibited the production of NO in activated BV2 microglial cells by $57 \pm 2 \%$ compared to vehicle in LPS treated controls $(p<0.0001)$ (Fig. 2). Similarly, all tested pyrrolobenzoxazepines inhibited NO release from activated BV2 microglia $(p<0.0001$ vs. control) (Fig. 3). Of note, 2, which showed the highest activity of the pyrrolobenzoxazepines tested here in regards to pregnenolone release (see Fig. 1), also showed the most potent inhibition of NO release, by $74 \pm 6 \%$ compared to vehicle in LPS treated controls $(p<0.0001)$. This was significantly more inhibition than

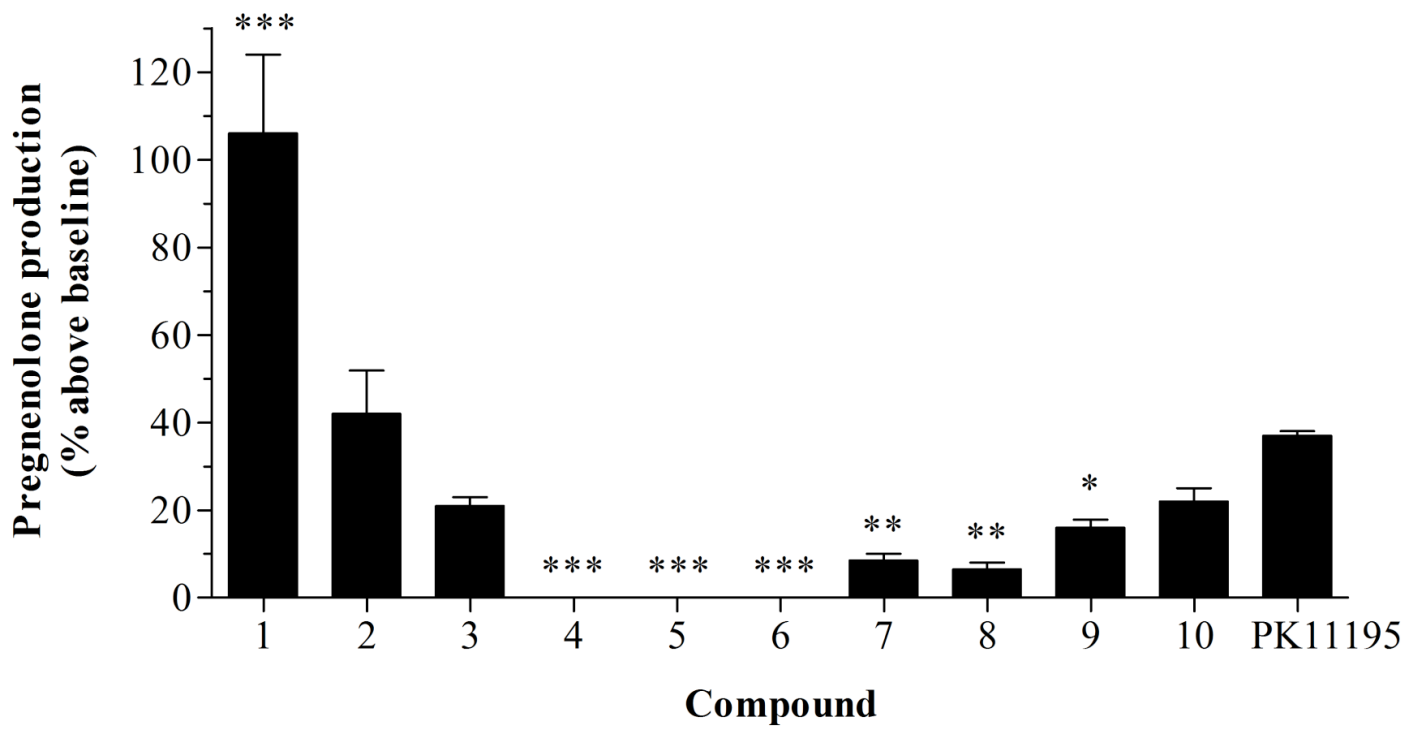

Fig. (1). Effect of $40 \mu \mathrm{M}$ ligands on pregnenolone release in rat C6 glioma cells. Experimental data represent the increase in pregnenolone production above baseline, denoted by mean \pm SEM of at least three independent experiments. ${ }^{*} p<0.05 ;{ }^{* *}$ $p<0.01 ;{ }^{* * *} p<0.001$, significantly different to PK 11195 using one-way ANOVA and Bonferroni's post-hoc test. 
that achieved with PK $11195(p<0.0001)$. Conversely, 5 that did not increase pregnenolone release above baseline (see Fig. 1), inhibited NO release by $66 \pm 3 \%$ compared to LPS treated control $(p<0.0001)$, and was therefore significantly more effective than PK 11195 $(p<0.01)$.

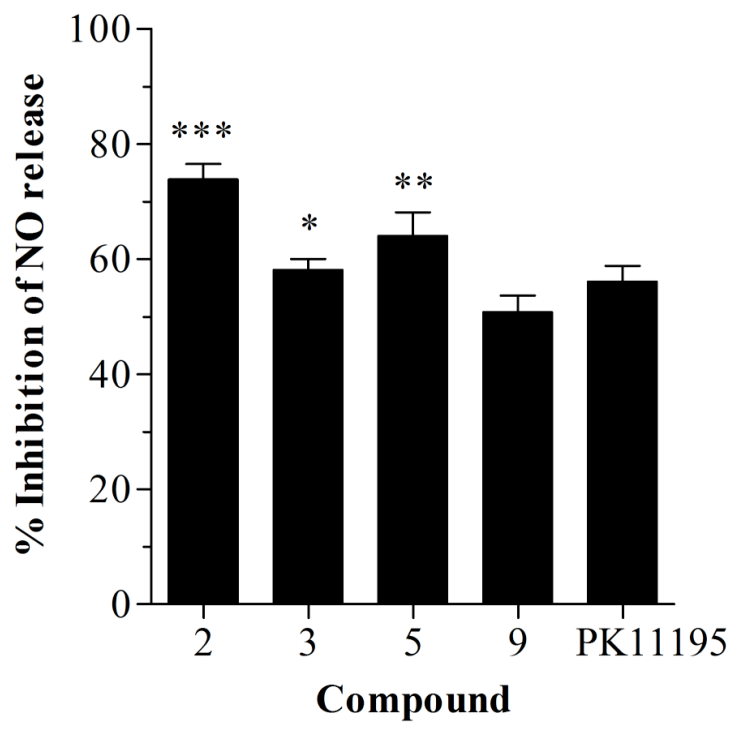

Fig. (2). Effect of selected pyrrolobenzoxazepines and PK $11195(40 \mu \mathrm{M})$ on NO production from activated BV2 microglial cells. Data are expressed as the percentage inhibition of NO production, compared to LPS-treated controls. Bars represent mean \pm SEM of at least 3 independent experiments carried out in duplicate. ${ }^{*} p<0.05$; ${ }^{* *} p<0.01$; ${ }^{* * *} p<0.001$, significantly different from PK 11195 in the presence of LPS, using one-way ANOVA and Bonferroni's post-hoc test. All compounds tested inhibited NO release to a level which was significantly different from vehicle alone in the presence of LPS $(p<0.001)$.

The mechanism by which TSPO ligands mediate their neuroprotective effects is proposed to be through the inhibition of microglial activation resulting in inhibition of cytokine and inflammatory mediator release [1]. Of interest, previous studies have correlated an increase in steroid synthesis with nerve regeneration [19-21] for both endogenous TSPO ligands $[19,20]$ and those tested in models of neuropathy in rats [20]. However, our reported inhibition of NO release occurred without any positive correlation to increases in pregnenolone release, suggesting that neuroprotective effects caused by the pyrrolobenzoxazepines occur independent of this mechanism. Our study may be limited by the mediator tested as an indicator of microglial activation, and future work should look to measure release of other cytokines from activated microglia.

TSPO ligands such as Ro 5-4864 and PK 11195 have anti-proliferative effects in various cancer cell lines, including the MCF-7 human breast cancer cell line [22]. Therefore, we also compared the antiproliferative effects of PK 11195 and 1-10 in MCF-7 cells using the sulforhodamine B assay. PK 11195 impaired the proliferation of MCF-7 cells by $54 \pm 3 \%$ compared to vehicle treated control (Fig. 3). Interestingly, all compounds showed anti-proliferative effects significantly different from the vehicle treated control ( $p<0.0001)$ (Fig. 3). However, compounds 1-8 and 10 were significantly better anti-proliferative agents when compared to PK 11195. Notably, compound 8 was the most potent inhibitor, reducing cell proliferation by $90 \pm 1 \%$ compared to controls, while having the lowest affinity of all the pyrrolobenzoxazepine ligands tested for human TSPO (Table 1). Thus, pyrrolobenzoxazepines showed anti-proliferative effects, however, of variable potency.

It has been previously suggested that antiproliferative effects caused by TSPO ligands may be mediated by other proteins within the cell [5]. Nevertheless, TSPO knockdown in MCF-7 breast cancer cells results in cell cycle arrest, and ultimately, reduced cell proliferation [23]. Thus, the

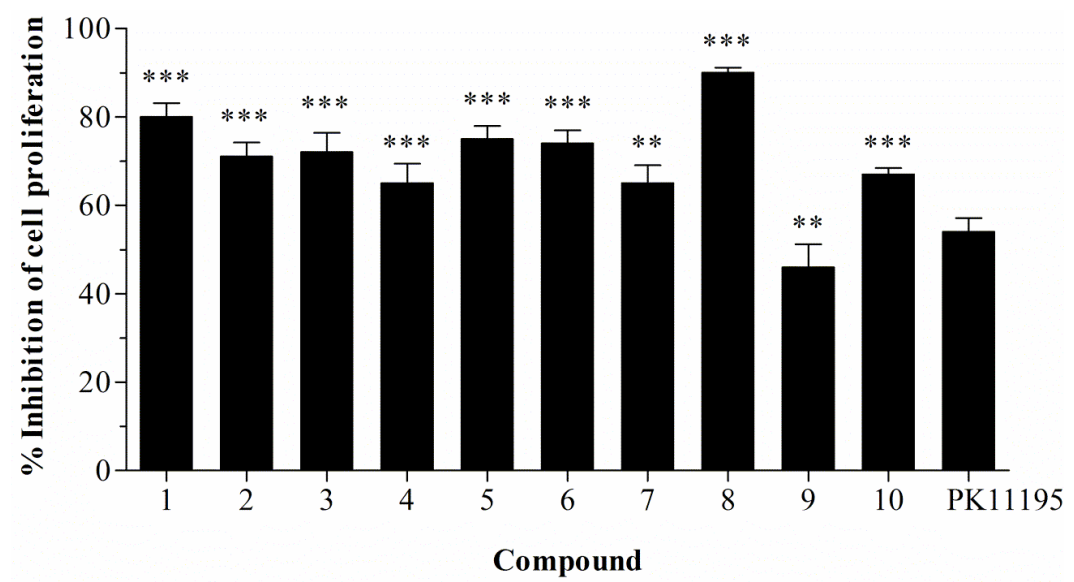

Fig. (3). Inhibition of cell proliferation in human MCF-7 breast cancer cells by $40 \mu \mathrm{M}$ pyrrolobenzoxazepines and PK 11195 . Experimental data represent mean \pm SEM of at least three independent experiments. ${ }^{* *} p<0.01 ;{ }^{* *} p<0.001$, significantly different from PK 11195 using one-way ANOVA and Bonferroni's post-hoc test. All ligands inhibited cell proliferation significantly better than the vehicle-treated control $(p<0.001)$. 
pyrrolobenzoxazepines which inhibit cell proliferation may act as TSPO antagonists, inhibiting TSPOmediated cell proliferation and growth. Alternatively, TSPO ligands may exert their anti-tumor effects by inducing apoptosis, as PK 11195 is able to induce mitochondrial release of cytochrome $c$, and ultimately cause mitochondrial uncoupling [23]. Pyrrolobenzoxazepines have been shown previously to reduce tumor cell growth in human cell lines through the induction of the caspase-3 family of proteases [5] and induction of $\mathrm{G}_{1}$ cell cycle arrest.

Despite the ability of the pyrrolobenzoxazepines to elicit a range of functional effects, no correlation between binding profile and the ability of the ligands to modulate pregnenolone and NO release, or the antiproliferative properties of the compounds was revealed. For example, compounds 5 and $\mathbf{6}$ were both high affinity ligands with a Hill slope of 1.0 , caused no stimulation of steroid synthesis, but inhibited cell proliferation to a degree comparable to 1-3, which increased steroid synthesis significantly and had Hill slopes of $0.99,0.75$, and 0.93 in rat, respectively. The absence of a correlation between binding affinity and functional profile has also been observed with other TSPO ligands [13, 18].

We propose that the lack of correlation between binding and functional assays may be partly attributed to the marked differences observed in ligand binding across species. However, the binding profile observed in rodent did not correlate to the functional assays in rodent cell lines measuring steroid production or NO release. Likewise, the binding profile in human did not correlate to the anti-proliferative effects observed in human MCF-7 breast cancer cells. Thus, while TSPO functional activity is traditionally denoted by pregnenolone production in rat C6 glioma cells at a ligand concentration of $40 \mu \mathrm{M}$, this may not be a true indication of solely TSPO-mediated effects. More recent studies suggest that testing ligands at nanomolar concentrations consistent with their binding affinities mediate very different functional effects compared to those assays performed at micromolar concentrations, whereby effects may be TSPOindependent [24]. Alternatively, recent evidence suggests that TSPO ligand binding varies across different tissues [25]. The existence of species differences also questions the validity of functional assays in rodent for predicting therapeutic outcomes relevant to human disease.

The current study provides evidence for a complex binding interaction at TSPO, and uncovers marked species differences between rat and human TSPO ligand binding, thus questioning the suitability of current binding and functional assays undertaken in rat tissues for the translation to human studies. While we have identified ligands with potential therapeutic effects, the lack of correlation to binding profile suggests currently accepted functional assays need to be optimized to take into account dose-related effects, and tissue and species disparity of binding sites. While the current
TSPO pharmacophore can assist in the development of selective high affinity ligands [1], there exists no consideration of binding site cooperativity or clear understanding of structural residues important for mediating downstream functional effects. The development of structure-activity relationships for TSPO ligand binding needs to take into account binding site cooperativity and the existence of species and tissue disparity, which will aid in the rational development of TSPO-based therapeutic drugs.

\section{ACKNOWLEDGEMENTS}

We wish to thank Dr. Katherine R. Leaver, Dr. Kylee M. Aumann, Kong Li, Dr. Ken Mewett and Dr. Hue Tran. This work was supported by funding from the National Health \& Medical Research Council (NHMRC) to L.M.I. and M.K.

\section{SUPPLEMENTARY MATERIAL}

Supplementary material is available on the publishers Web site along with the published article.

\section{REFERENCES}

[1] Scarf AM, Ittner LM, Kassiou M. The translocator protein (18 $\mathrm{kDa}$ ): Central nervous system disease and drug design. J Med Chem 2009; 52: 581-92.

[2] Scarf AM, Kassiou M. The translocator protein. J Nucl Med 2011; 52: 677-80

[3] Hardwick M, Fertikh D, Culty M, Li H, Vidic B, Papadopoulos V. Peripheral-type benzodiazepine receptor (PBR) in human breast cancer: Correlation of breast cancer cell aggressive phenotype with PBR expression, nuclear localization, and PBR-mediated cell proliferation and nuclear transport of cholesterol. Cancer Res 1999; 59: 831-42.

[4] Wilms H, Claasen J, Rohl C, Sievers J, Deuschl G, Lucius R. Involvement of benzodiazepine receptors in neuroinflammatory and neurodegenerative diseases: evidence from activated microglial cells in vitro. Neurobiol Dis 2003; 14: 417-24.

[5] Zisterer DM, Campiani G, Nacci V, Williams DC. Pyrrolo-1,5benzoxazepines induce apoptosis in HL-60, Jurkat, and Hut78 cells: A new class of apoptotic agents. J Pharmacol Exp Ther 2000; 293: 48-59.

[6] Carmel I, Fares FA, Leschiner S, Scherübl H, Weisinger G, Gavish M. Peripheral-type benzodiazepine receptors in the regulation of proliferation of MCF-7 human breast carcinoma cell line. Biochem Pharmacol 1999; 58: 273-8.

[7] Le Fur G, Guilloux F, Rufat $P$, et al. Peripheral benzodiazepine binding sites: effect of PK 11195, 1-(2chlorophenyl)-N-methyl-(1-methylpropyl)-3 isoquinolinecarboxamide. II. In vivo studies. Life Sci 1983. 32: 1849-56.

[8] Le Fur G, Vaucher N, Perrier M, et al. Differentiation between two ligands for peripheral benzodiazepine binding sites, $\left[{ }^{3} \mathrm{H}\right] \mathrm{Ro5}-4864$ and $\left.{ }^{3} \mathrm{H}\right] \mathrm{PK} 11195$, by thermodynamic studies. Life Sci 1983; 33: 449-57.

[9] Marangos PJ, Patel J, Boulenger JP, Clark-Rosenberg R. Characterization of peripheral-type benzodiazepine binding sites in brain using $\left.{ }^{3} \mathrm{H}\right] \mathrm{Ro}$ 5-4864. Mol Pharmacol 1982; 22: 26-32.

[10] Rao VLR, Butterworth RF. Characterization of binding sites for the o3 receptor ligands $\left[{ }^{3} \mathrm{H}\right] \mathrm{PK} 11195$ and $\left[{ }^{3} \mathrm{H}\right] \mathrm{R}$ o5-4864 in human brain. Eur J Pharmacol 1997; 340: 89-99.

[11] Awad M, Gavish M. Binding of $\left[{ }^{3} \mathrm{H}\right] \mathrm{Ro} 5-4864$ and $\left[{ }^{3} \mathrm{H}\right] \mathrm{PK}$ 11195 to cerebral cortex and peripheral tissues of various 
species: Species differences and heterogeneity in peripheral benzodiazepine binding sites. J Neurochem 1987; 49: 140714.

[12] Farges R, Joseph-Liauzun E, Shire D, Caput D, Le Fur G, Ferrara $P$. Site-directed mutagenesis of the peripheral benzodiazepine receptor: identification of amino acids implicated in the binding site of Ro5- 4864. Mol Pharmacol 1994; 46: 1160-7.

[13] Campiani G, Nacci V, Fiorini I, et al. Synthesis, biological activity, and SARs of pyrrolobenzoxazepine derivatives, a new class of specific peripheral-type benzodiazepine receptor ligands. J Med Chem 1996; 39: 3435-50.

[14] Awad M, Gavish M. Peripheral-type benzodiazepine receptors in human cerebral cortex, kidney, and colon. Life Sci 1991; 49: 1155-61.

[15] Wendler G, Lindemann P, Lacapère J-J, Papadopoulos V. Protoporphyrin IX binding and transport by recombinant mouse PBR. Biochem Biophys Res Commun 2003; 311: 847-52.

[16] Yeliseev AA, Kaplan S. TspO of rhodobacter sphaeroides. A structural and functional model for the mammalian peripheral benzodiazepine receptor. J Biol Chem 2000; 275: 5657-67.

[17] Papadopoulos V, Brown AS. Role of the peripheral-type benzodiazepine receptor and the polypeptide diazepam binding inhibitor in steroidogenesis. J Steroid Biochem Mol Biol 1995; 53: 103-10.

[18] James ML, Fulton RR, Vercoullie J, et al. DPA-714, a new translocator protein-specific ligand: Synthesis, radiofluorination, and pharmacologic characterization. J Nucl Med 2008; 49: 814-22.

[19] Lacor P, Benavides J, Ferzaz B. Enhanced expression of the peripheral benzodiazepine receptor (PBR) and its endogenous ligand octadecaneuropeptide (ODN) in the regenerating adult rat sciatic nerve. Neurosci Lett 1996; 220: 61-5.

[20] Lacor P, Gandolfo P, Tonon M-C, et al. Regulation of the expression of peripheral benzodiazepine receptors and their endogenous ligands during rat sciatic nerve degeneration and regeneration: a role for PBR in neurosteroidogenesis. Brain Res 1999; 815: 70-80.

[21] Koenig $\mathrm{H}$, Schumacher M, Ferzaz B et al. Progesterone synthesis and myelin formation by Schwann cells. Obstet Gynecol Surv 1995; 50: 792-3.

[22] Hunakova L, Bodo J, Chovancova J, Sulikova G Pastorekova S, Sedlak J. Expression of new prognostic markers, peripheral-type benzodiazepine receptor and carbonic anhydrase IX, in human breast and ovarian carcinoma cell lines. Neoplasma 2007; 54: 541-8.

[23] Li W, Hardwick MJ, Rosenthal D, Culty M, Papadopoulos V. Peripheral-type benzodiazepine receptor overexpression and knockdown in human breast cancer cells indicate its prominent role in tumor cell proliferation. Biochem Pharmacol 2007; 73: 491-503.

[24] Choi J, Ifuku M, Noda M, Guilarte TR. Translocator protein (18 kDa)/peripheral benzodiazepine receptor specific ligands induce microglia functions consistent with an activated state. Glia 2011; 59: 219-30.

[25] Kreisl WC, Fujita M, Fujimura $\mathrm{Y}$, et al. Comparison of $\left[{ }^{11} \mathrm{C}\right]$ (R)-PK 11195 and $\left[{ }^{11} \mathrm{C}\right] \mathrm{PBR} 28$, two radioligands for translocator protein $(18 \mathrm{kDa})$ in human and monkey: Implications for positron emission tomographic imaging of this inflammation biomarker. Neuroimage 2010; 49: 2924-32. 